\title{
HWS - Kraniozervikale Übergangsstörungen Wiederherherstellende Verfahren bei gestörten Funktionen der HWS und des kraniozervikalen Überganges
}

\section{Inhaltsverzeichnis}

1 Einleitung ...262

2 Reversible Funktionsstörungen von Gelenken, Muskeln und Bändern und deren Behandlung ...263

2.1 Funktionsstörungen der Wirbelgelenke und des Kopfgelenks... 263

2.1.1 Manualtherapeutische Verfahren ...264

2.2 Funktionsstörungen des Muskel- und Bandapparates sowie der Weichteile und deren Behandlung... 264

2.2.1 Physikalische Verfahren...265

2.2.2 Medikamentöse Behandlung/Schmerztherapie ... 265

2.3 Medizinische Trainingstherapie zur Sicherung des Langzeittherapieerfolges...266

2.4 Begleitende Therapiemaßnahmen ... 266

3 Strukturell bedingte, angeborene oder erworbene Funktionsstörungen der HWS und des kraniozervikalen Übergangs ...266

3.1 Posttraumatische Störungen der Wirbelsäule, des Kopfgelenkes, des Weichteil- und Bandapparates, der Bandscheiben und des Rückenmarkes ... 267

3.1.1 Operative Therapie...267

3.1.2 Konservative Therapie... 268

3.2 Folgezustände nach tumorchirurgischen Eingriffen oder iatrogene Störungen der HWS und des kraniozervikalen Übergangs ... 269

3.3 Angeborene, chronisch-degenerative und andere Funktionsstörungen der HWS und des kraniozervikalen Übergangs ... 270

4 Rationale Therapieplanung nach Leitsymptomen ... 270

Literatur (Hinweis: erscheint nur in der Online-Ausgabe)

\section{Zusammenfassung}

Die Halswirbelsäule (HWS) ist der beweglichste Teil der Wirbelsäule und hat damit auch das größte Störungs- bzw. Verletzungspotenzial. Während andere Wirbelsäulenabschnitte (z.B. die Lendenwirbelsäule) primär durch ihren Aufbau eine knöcherne Führung aufweisen, ist die HWS muskel- und bandgeführt. Evolutionsbiologisch ist dies zur freien Orientierung des Kopfes und der Sinnesorgane im Raum erforderlich. Durch die vielfältigen muskulären, neuronalen und funktionellen Verknüpfungen der HWS zum Kauapparat, durch Projektionen in den Hirnstamm, durch die Head'schen Zonen, die schmerzbestimmende Gammaschleife des Rückenmarkes („spinales Gedächtnis“) können bunte klinische Bilder bei Störungen der HWS auftreten. Leitsymptome sind dabei Schmerz (mit Projektionen in das Ohr, die Kiefergelenksregion, den Hinterkopf u.a.), Unsicherheitsgefühl, Globusgefühl und Störungen der Sensorik (Hören und Sehen). Neben funktionellen Störungen spielen Unfallfolgezustände, degenerative oder immunologisch vermittelte Erkrankungen die größte Rolle. Konservative Therapieverfahren (Physiotherapie, medikamentöse Therapie) haben ebenso wie die neurochirurgischen Interventionen an der HWS und im kraniozervikalen Übergang den wichtigsten Stellenwert innerhalb der Therapiekonzepte, die möglichst breit und interdisziplinär (Orthopäden, Unfallchirurgen, Neurologen, Physiotherapeuten, HNO) angelegt sein sollten.

\section{Schlüsselwörter}

HWS · kraniozervikaler Übergang · Physiotherapie · whiplash · Bandscheibe $\cdot$ Spinalchirurgie 


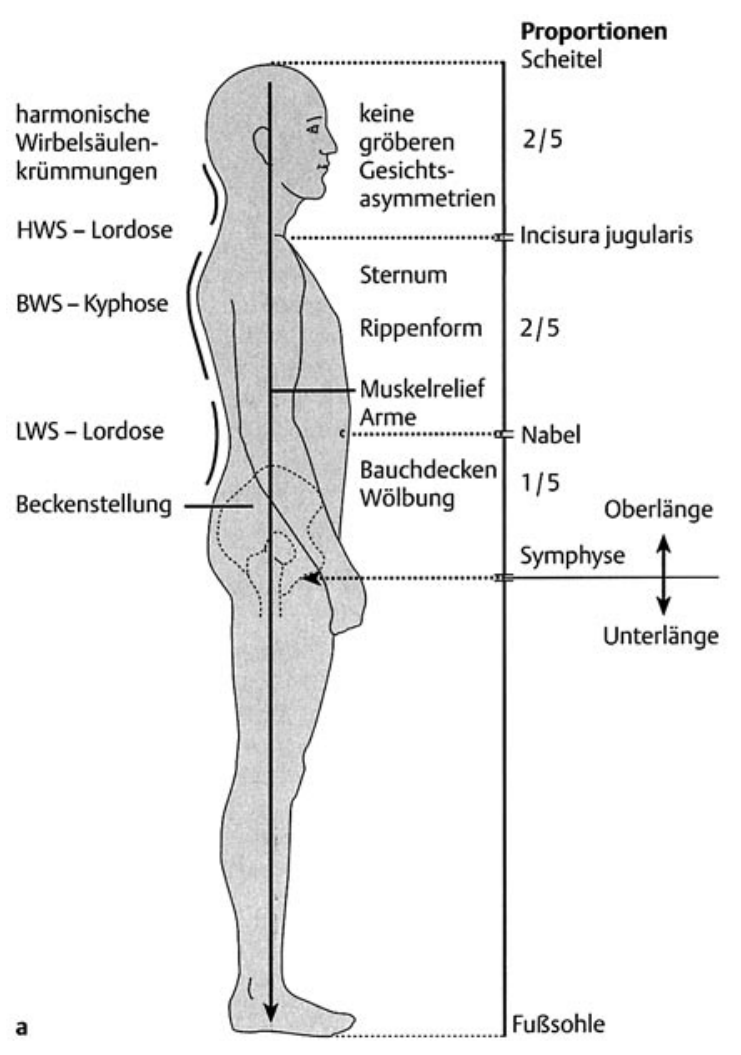

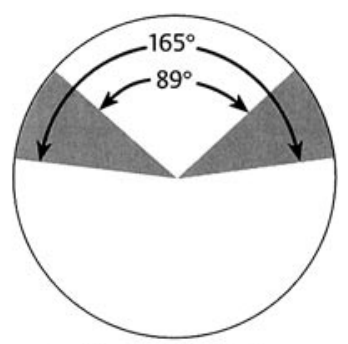

A Flexion/Extension

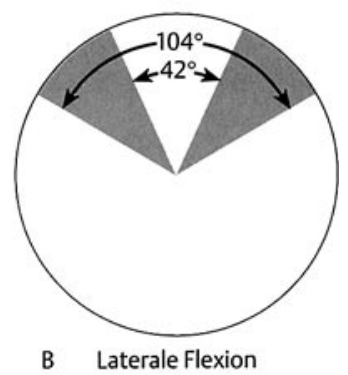

B Laterale Flexion

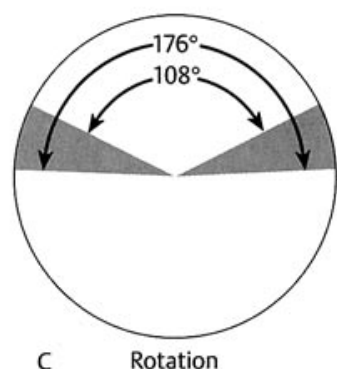

Abb. 1 a Seitliche Gesamtinspektion der Wirbelsäule mit Proportionen und physiologischen Krümmungen. b Normales Ausmaß der möglichen Globalbewegungen der HWS (A - Ante- und Retroflexion, B - Lateroflexion, C - Rotation). c Höchstmögliche Anzahl an Freiheitsgraden der HWS. Aus: Ernst A, Meyer-Holz J, Weller E. Manuelle Medizin an der Halswirbelsäule. Stuttgart, New York: Georg Thieme, 1998: 6, Abb. 3a, b, c.
Die Halswirbelsäule (HWS) sowie der kraniozervikale Übergang (KZÜ) teilt man in drei funktionell eigenständige Abschnitte ein, die Kopfgelenke (Kondylen der Hinterhauptsschuppe, Atlas-Axis), die mittlere HWS (dritter bis fünfter Halswirbel) sowie die untere HWS und den zervikothorakalen Übergang (sechster und siebenter Halswirbel, erster bis dritter Brustwirbel, Rippenansätze) (Abb.1).

Der Kopfgelenksbereich stellt dabei einen physiologisch und anatomisch besonderen Abschnitt dar, da er vielfältige neurophysiologische Verbindungen [1] mit einem hohen Maß an Beweglichkeit kombiniert (Abb.1). Diese Besonderheiten sind phy- logenetisch bedingt [2] und machen die besondere Stellung dieser kraniozervikalen Übergangsregion deutlich [zur Übersicht vgl. 3, 4]. Auf diese Weise ist die HWS prädestiniert für segmentale Bewegungsstörungen, da sie der beweglichste (höchstmögliche Anzahl an Freiheitsgraden der Bewegung bei geringster ossärer, ligamentärer bzw. muskulärer Führung), aber auch der störanfälligste Abschnitt der Wirbelsäule ist. So wird auch verständlich, warum Störungen in anderen Wirbelsäulenabschnitten (z.B. Beinlängenverkürzung, Blockierungen im Ileosakralgelenk, Skoliosen, Lumboischialgien) den Bereich der HWS beeinflussen können, um z.B. funktionelle Störungen in solch einem entfernten Bewegungssegment („Verkettungen“) zu bewirken [5]. Die Bewegungen unseres Achsenorgans unterliegen einer zentralen Steuerung und sind rückgekoppelt. Ein Bewegungsentwurf wird 
vom Kleinhirn und den Stammganglien kontrolliert und modifiziert und unterliegt vielfachen (z.B. emotionalen, sympathischen, sensorisch-integrativen) Einflüssen [4]. Bei der Umsetzung des Entwurfes in der Peripherie ist vorwiegend die Muskulatur beteiligt, die eine überragende funktionelle Rolle an der HWS bei der Ausformung des Bewegungsentwurfes spielt und im Falle einer pathophysiologischen Störung (,Schmerz“) durch die $\gamma$-Schleife des Rückenmarks determiniert wird [6].

Ziel der vorliegenden Übersicht soll es sein, die spezifischen Funktionsstörungen der HWS und ihre Therapie- und Rehabilitationsmöglichkeiten aufzuzeigen. Während bei den knöchernen Veränderungen (z. B. Frakturen, Deformitäten) chirurgische Verfahren im Vordergrund stehen, spielen konservativ-manualmedizinische Verfahren bei Störungen der Muskulatur und der Weichteile der HWS die wichtigste therapeutisch-rehabilitative Rolle (Chirotherapie, Krankengymnastik, Vojta-Therapie, Osteopathie, physikalische Therapie). Auch Funktionsverluste (z.B. Querschnittslähmung oder Folgezustände komplexer Traumen) sind kompensatorisch (aber nicht vollständig wiederherstellend!) am besten mit Hilfe konservativ-manualmedizinischer Verfahren behandelbar (neuronale Plastizität !) [7]. Ein wichtiger Schwerpunkt wissenschaftlicher Untersuchungen von chronifizierten Verletzungen an der HWS (z.B. „whiplash-associated disorders", WAD) [8] waren die sekundären psychosomatischpsychiatrischen, assoziierten Störungen $[9,10]$ infolge gestörter zerebraler Verarbeitung [11] aufgrund des Dauerschmerzes [12] bei solchen chronischen HWS-Funktionsstörungen. Hier konnten zugrundeliegende, organische Korrelate (Neurotransmitterstörungen, zerebrale Perfusionsstörungen, kognitiv-mnestische Verarbeitungsstörungen) identifiziert werden, die aus therapeutischer Sicht für begleitende Therapiemaßnahmen bedeutsam sind [3,13 - 16]. Funktionsstörungen der HWS der ersten Lebensjahre nehmen einen immer breiteren Raum in Schrifttum ein (z.B. KISS-Syndrom, sensomotorische Integrationsstörungen) [z.B. 17-19], so dass die vorliegende Arbeit aus Platzgründen sich vorwiegend auf den adulten Bereich bezieht. Sofern jedoch Störungen aus den ersten Lebensjahren klinisch-relevant „herüberragen“ (z. B. Skoliose, Hüftdysplasie), finden sie Beachtung.

In der Bundesrepublik Deutschland sucht jeder fünfte Patient einer allgemeinärztlichen Praxis und jeder dritte Patient einer orthopädischen Praxis diese wegen „Rückenschmerzen“ auf, die damit die Hauptursache von Arbeitsunfähigkeitszeiten und Krankenhausaufenthalten bilden [20]. Die Halswirbelsäule wird dabei gleich nach der Lendenwirbelsäule (Lumboischialgie) als Hauptort der Störung identifiziert. Die Leistungsaufwendungen deutscher Krankenversicherer betrugen für die Behandlungen solcher Erkrankungen 199918 Mrd. €, was die sozioepidemiologische und finanzielle Dimension dieser Erkrankungen verdeutlicht [20].

\section{Reversible Funktionsstörungen von Gelenken, Muskeln, Bändern und deren Behandlung}

Das der Manuellen Medizin zugrunde liegende Denkmodell basiert auf der Vorstellung, dass Störungen der Gelenkbeweglichkeit zu Störungen im „Bewegungssegement“ (,Arthron“ oder „Vertebron“) (Abb. 2), d. h. dem zugehörigen Muskel-, Faszien-, Band-

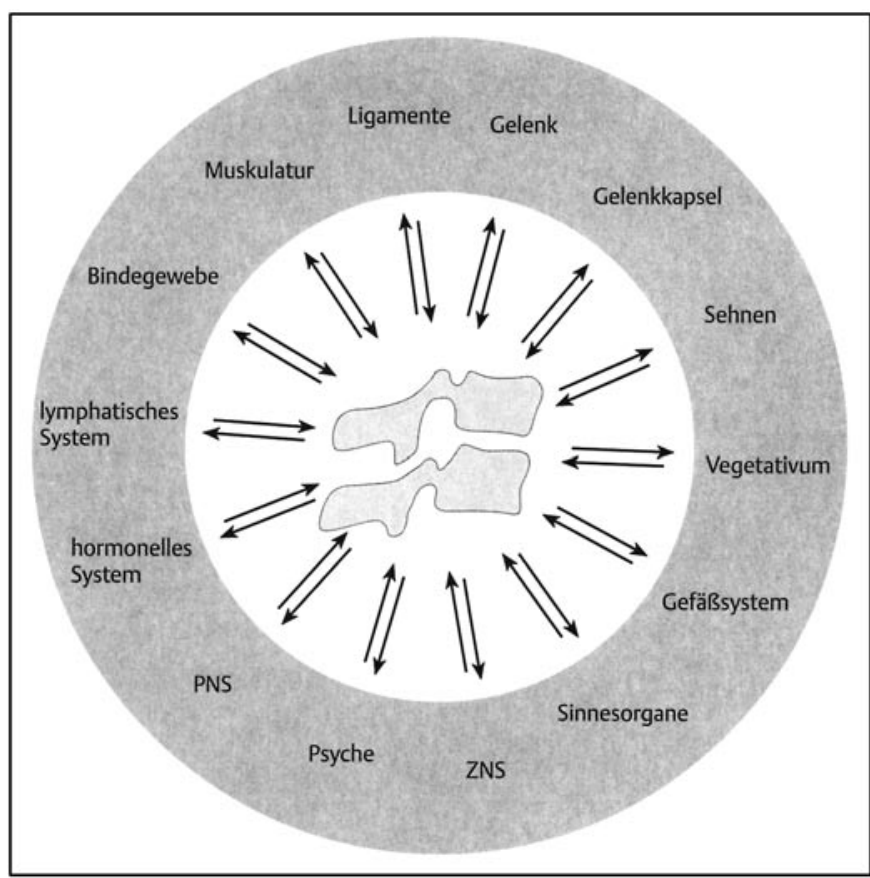

Abb. 2 Schematische Verknüpfung der Elemente des „Bewegungssegments" mit den einzelnen Organsystemen, aus: Ernst A, MeyerHolz J, Weller E. Manuelle Medizin an der Halswirbelsäule. Stuttgart, New York: Georg Thieme, 1998: 3, Abb. 2.

und Hautbestandteil (und umgekehrt) führen können [4]. Entsprechend dieses neurophysiologischen Ansatzes sind die einzelnen Wirbelgelenke (ihre Proprio- bzw. Nozizeptoren), die Muskulatur, Bandscheiben und der Bandapparat sowie das zugehörige Hautsegment über vielfache reflektorische Vorgänge miteinander verknüpft (Abb.3). Die reversible, hypomobile Dysfunktion eines Gelenks („Blockierung“) spielt als Pathomechanismus von Funktionsstörungen der Wirbelsäule die wichtigste Rolle [5] (Abb.4). Sie ist mit einem partiellen Verlust an Mobilität einerseits („gelenkmechanischer Aspekt“), als auch mit neurophysiologisch gesteuerten Begleitreaktionen verknüpft (,neurologischer Aspekt“), wie z.B. Haut-, Muskelschmerz, Organstörung [4]. Da Manualtherapie (Synonym: Chirotherapie) Reflextherapie ist, sollten die Therapieformen in aufsteigender Intensität angewandt werden: Weichteiltechniken-Muskelrelaxationstechniken-Mobilisationen-Manipulationen [21]. Allgemein anerkannt ist das Paradigma, dass Befunde an der Haut primär über die Haut (z.B. Dermatomirritation der Head'schen Zone), Befunde am Muskel über die Muskulatur (z.B. Myalgien, Myogelosen) und Befunde am Gelenk über das Gelenk (z.B. Gelenkblockaden) therapiert werden [22].

\subsection{Funktionsstörungen der Wirbelgelenke und des Kopfgelenkes}

Die Gelenkblockierung einzelner Wirbelgelenke der HWS ist die am häufigsten vorkommende, akute Funktionsstörung der Wirbelsäule mit der höchsten klinischen Relevanz [2]. Sie entsteht typisch durch statische Fehlbelastung (akute und chronische Fehlbelastungen, z. B. PC-Arbeit) bzw. durch Überlastung (z. B. ÜberKopf-Arbeit, Gelenkblockierung in anderen Wirbelsäulengelenken mit Verkettung, z.B. nach schwerem Heben/Tragen). Die Auswahl der einzusetzenden manualtherapeutischen Verfahren zur Behandlung erfolgt nach dem jeweiligen Stadium der Aktua- 


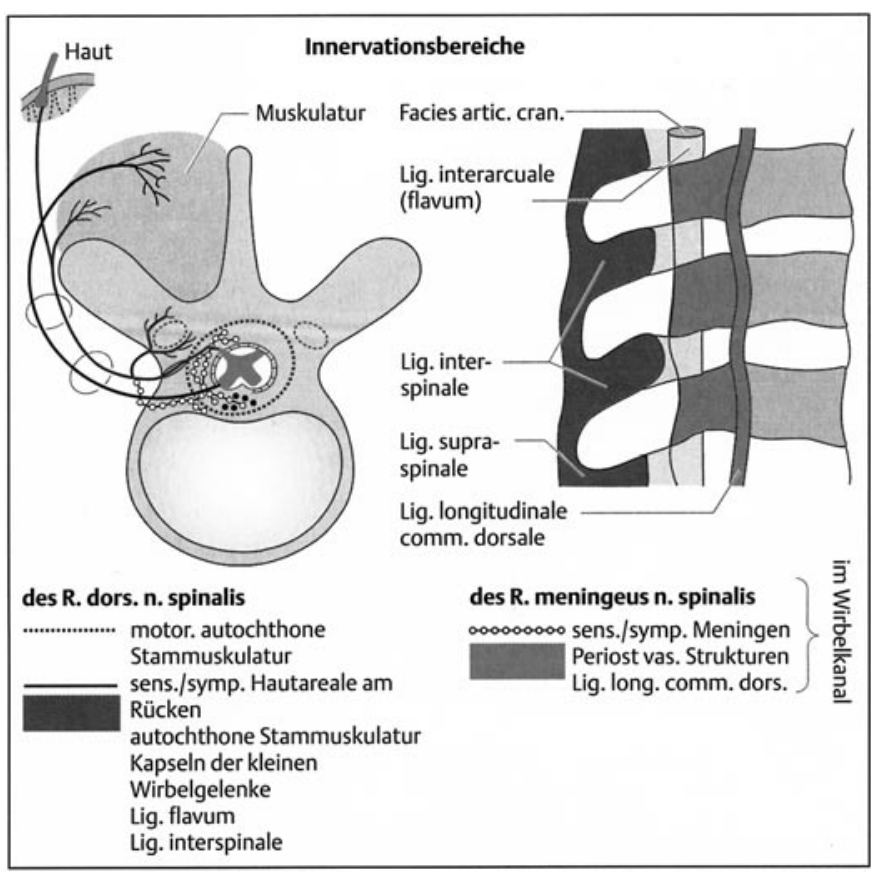

Abb. 3 Neurale Versorgung des Bewegungssegments und reflektorische Verknüpfungen zwischen Wirbelgelenk und zugehörigem Muskelbzw. Hautgebiet („Bewegungssegment“), aus: Ernst A, Meyer-Holz J, Weller E. Manuelle Medizin an der Halswirbelsäule. Stuttgart, New York: Georg Thieme, 1998: 3, Abb. 1.

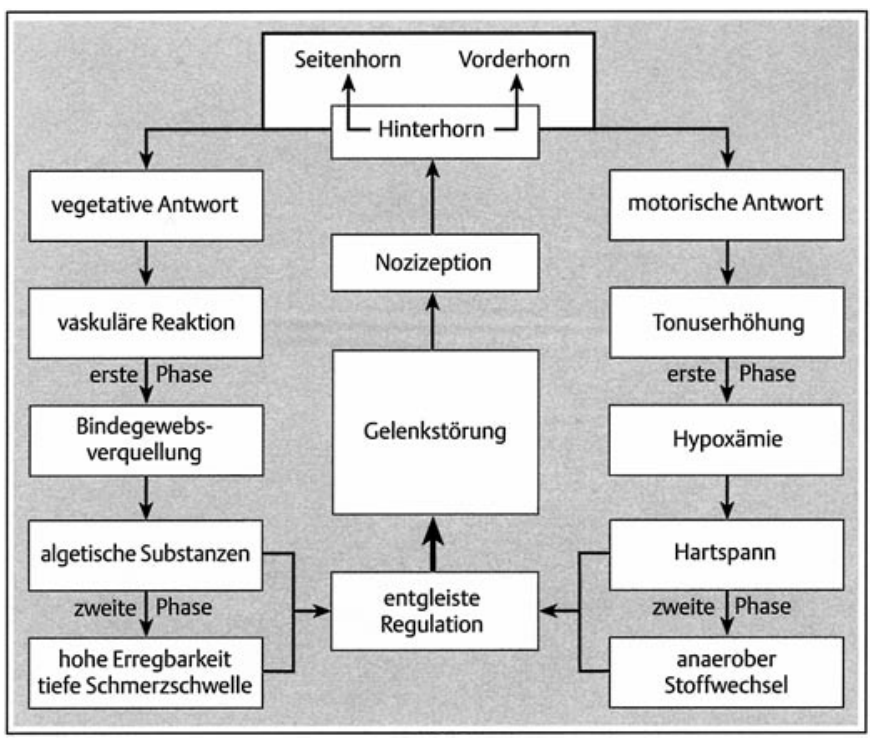

Abb. 4 Entstehungsmechanismus der motorischen und vegetativen Nozireaktion bei gestörter Gelenkbeweglichkeit, aus: Ernst A, MeyerHolz J, Weller E. Manuelle Medizin an der Halswirbelsäule. Stuttgart, New York: Georg Thieme, 1998: 13, Abb. 7.

lität der Erkrankung (z. B. akut/hochakut, subakut, chronisch). In erster Linie lassen sich durch manipulative Techniken (Manipulation mit Impuls) die Gelenkblockierungen am schnellsten und effektivsten lösen, wenn keine Kontraindikationen bestehen (z.B. massive, begleitende, schmerzhafte Muskelverspannung oder allgemeine Hypermobilität aller Gelenke) [22]. Im ersten Fall sollte eine passive Mobilisationsbehandlung zur Anwendung kommen oder man sollte medikamentös für einige Tage vorbehandeln (vgl. 2.2.2). Hypermobile Patienten sollten möglichst nur jenseits der betroffenen Gelenke behandelt werden, um der habituellen Hypermobilität nicht Vorschub zu leisten. Ein Muskelaufbau (vgl. 2.3) ist dringend anzuraten [23,24].

\subsubsection{Manualtherapeutische Verfahren}

Es ist das Ziel der Manipulationsbehandlung am Gelenk, mit einem Handgriff (low-amplitude-high-velocity-Technik) eine Blockierung zu beheben, um so das joint play wieder herzustellen, wobei verschiedene Techniken für die einzelnen Gelenke der HWS zur Anwendung kommen [5,21,22].

Mobilisationsbehandlungen von Wirbelsäulengelenken sind in alle Bewegungsrichtungen möglich. Dabei sind die Gelenktraktionen die wirksamsten Mobilisationsformen. Diese Traktionen sind am effektivsten in der Ruhelage des Gelenkes durchzuführen, also am liegenden Patienten. Da die Flexionsbewegungen nach ventral (Divergenzbewegung) bzw. dorsal (Konvergenzbewegung) Gleitbewegungen darstellen, muss man zusätzlich zu der mobilisierenden Gleitbewegung in Konvergenz noch eine Traktion (Pikkolotraktion) anwenden. Mobilisationen haben zum Ziel, insbesondere bei schmerzhaft eingeschränkter Gelenkbeweglichkeit, eine Vergrößerung des Bewegungsspielraums durch passive, wiederholte Traktionen oder Gleitbewegungen mit geringer Geschwindigkeit und zunehmend vergrößerter Amplitude zu erzielen. Eine klassische Möglichkeit passiver Mobilisationsbehandlung im Bereich der HWS ist die axiale Traktion zur Entlastung eines oder mehrerer Segmente gleichzeitig [5]. Die passiven Mobilisationstechniken können mit Weichteiltechniken kombiniert werden. Häufig werden hierbei die Handlungen des Therapeuten durch die bahnende Mithilfe (Fazilitation) des Patienten (z. B. Mobilisation in der Exspiration, Einbeziehung der Blickwendung des Patienten) begleitet (vgl. 2.4).

Die aktive Mobilisationsbehandlung dient der Behandlung von Gelenkblockierungen durch Behandlung der zum jeweiligen Bewegungssegment gehörigen Muskulatur. Sie umfasst neben der Muskelenergietechnik (Mobilisationskraft durch den Patienten) auch Bahnungs- und Automobilisation (Blickwende- bzw. Atemtechnik). Die postisometrische Relaxation wird analog - unter Berücksichtigung schulenspezifischer Unterschiede, Besonderheiten und Modifikationen - auch als Muskelenergietechnik bzw. als neuromuskuläre Technik bezeichnet [25]. Dabei darf die Mobilisation jeweils nur in die schmerzfreie Richtung erfolgen und nur wenige Sekunden andauern, um die physiologische Bewegungsgrenze des Gelenkes nicht zu überschreiten [6].

\subsection{Funktionsstörungen des Muskel- und Bandapparates sowie der Weichteile und deren Behandlung}

Die akute Funktionsstörung des Muskel- und Bandapparates (akute Myalgie, Myogelose, Myotendinose) entsteht durch mechanische („Überdehnung“) oder physikalische Einwirkung („Kälte“) und bildet sich klinisch als „Schiefhals“ oder „,akutes Zervikalsyndrom" (hochgradige Bewegungseinschränkung der HWS in allen Richtungen) ab (Abb. 3). Zur Schmerzreduktion und zur Bahnung des Therapiebeginnes sollte initial eine Schmerzbehandlung durch therapeutische Lokalanästhesie (TLA), ggf. begleitet durch systemische Medikamentengabe (vgl. 2.2.2) und Orthesenverordnung (z.B. für 12-24 h) erfolgen. Wenn dann eine Behandlung möglich wird, eignen sich am besten zu Beginn Weichteiltechniken [6]. Sie therapieren primär an der Muskulatur und be- 
wirken damit indirekt eine Lösung und Lockerung der darüberliegenden, veränderten Bindegewebsstruktur. Die Hauptaufgabe der Weichteiltechniken liegt somit in der Entspannung der Muskulatur durch Quer- und Längsdehnungen. Damit finden sie ebenso wie die Mobilisation - vor allem Anwendung an der schmerzhaft verspannten und verkürzten Muskulatur und ihrem zugehörigen, pathologisch veränderten Bewegungssegment. Dieses Therapieprinzip lässt sich wirkungsvoll durch die (später einsetzende) Kombination mit der Mobilisationsbehandlung (2.1) unterstützen. Zu den Weichteiltechniken gehören die Inhibitionstechnik, die tiefe Quermassage (deep friction nach Cyriax) sowie die Dehnungstechnik [5].

Bei den akuten Schmerzzuständen an der HWS („akutes Zervikalsyndrom", akute HWS-Weichteildistorsion), die sich sowohl durch multiple Gelenkblockaden, eine begleitende Muskelabwehrspannung und Schmerzen im zugehörigen Dermatom auszeichnen, sind osteopathische Verfahren (z.B. Kraniosakraltherapie) zur Detonisierung der Muskulatur und Wiederherstellung der normalen Gewebstextur gut anwendbar [26]. Besonders in der hochakuten Phase gelingt eine klassisch-manualmedizinische Behandlung dieser stark schmerzhaften Regionen ansonsten kaum $[25,26]$.

\subsubsection{Physikalische Verfahren}

Der Einsatz physikalischer Verfahren hat in den letzten Jahren im klinischen Alltag deutlich abgenommen. Die Thermo-, Elektround Ultraschalltherapie haben aber noch ihren festen Platz in der Behandlung akuter Erkrankungen und deren Rehabilitation [27]. Akute (und ein Teil der chronisch-rheumatischen) Störungen des Muskel- und Weichteilmantels der HWS sollten durch Wärmeentzug behandelt werden (Erregung der kutanen Kaltrezeptoren führt zu einer Afferenzstimulation mit einer Aktivierung der spinalen Eingangskontrolle und sekundärer Schmerzreduktion) [28]. In der chronischen Phase der Erkrankung empfiehlt sich Wärmezufuhr (zur Verbesserung der Elastizität und Reduktion der Viskosität) [28].

In der Anwendung der Elektrotherapie hat die Galvanisation durch Gleichstrom bei subakuten Myotendinosen und Arthropathien (z.B. nach Bagatelltraumen) ihre Berechtigung. Andere Therapieverfahren (Wechselströme, Ultraschall) sind in der Anwendung an der HWS selten.

\subsubsection{Medikamentöse Behandlung/Schmerztherapie}

Der Schmerz tritt bei der Gelenkblockierung immer dann auf, wenn zusätzliche nozizeptive Afferenzen aus den Gelenkkapseln aktiviert werden. Die nozizeptive Reaktion erfolgt umgehend über die Muskulatur so dass durch eine reflektorische Verspannung eine Ruhigstellung des betroffenen Gelenks erreicht werden soll [4,29]. Bei aktiver Bewegung empfindet dies der Patient als Schmerz. Dabei muss dieser Schmerz nicht unbedingt in unmittelbarer, direkter Nachbarschaft des funktionsgestörten Gelenkes empfunden werden, sondern kann fortgeleitet (im Muskel, dem Dermatom) auftreten. Die Schmerztherapie bei Gelenkund auch bei Muskel/Weichteilerkrankungen der HWS hat in den letzten Jahren eine deutliche Aufwertung im therapeutischen Repertoire erfahren, da man beschreiben konnte, dass sofortige Schmerztherapie bei der Akutverletzung die Gefahr der Chronifizierung reduziert und dass sekundäre psychische Fehlent-

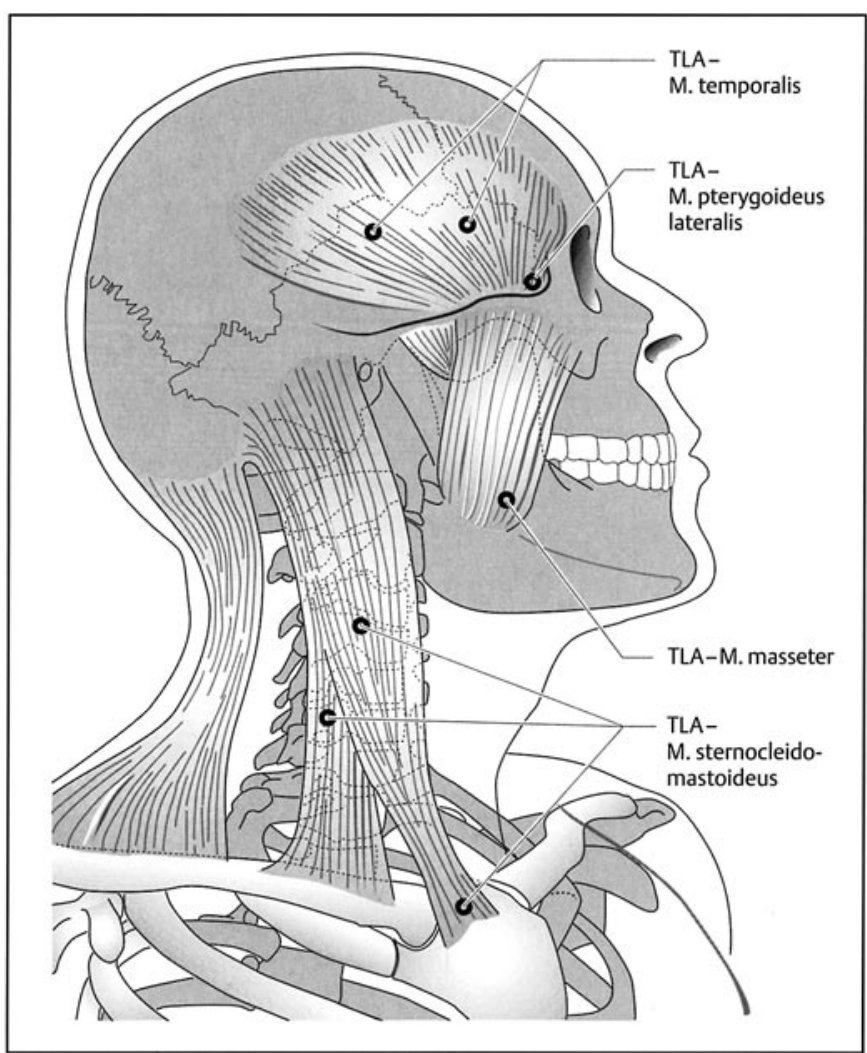

Abb. 5 Typische Injektionsorte zur Therapie über die Muskulatur des Kopf-Hals-Gebietes (TLA), aus: Ernst A, Meyer-Holz J, Weller E. Manuelle Medizin an der Halswirbelsäule. Stuttgart, New York: Georg Thieme, 1998: 53, Abb. 48.

wicklungen (PTBS, sekundäre, reaktive Depression, somatoforme Schmerzstörungen, Angst- und Anpassungsstörung) so am effizientesten verhindert werden können [11,12,30]. Ohne schmerzausschaltende Therapie bei der chronischen Funktionsstörung der HWS sind keine begleitenden Therapiemaßnahmen erfolgversprechend anwendbar (Manual/Physio/Ergotherapie, Hirnleistungstraining) [31]. Der unzureichend therapierte Schmerz ist somit der Hauptprädiktor für einen schlechten Outcome und für die sekundäre, psychische Fehlentwicklung nach HWS-Traumen $[14,32]$.

Die therapeutische Lokalanästhesie (TLA) ist eine Form der Neuraltherapie [33]. Die schmerzauslösende Struktur (Myotendinosen, z.B. paravertebrale Triggerpunkte, M. levator scapulae, M. sternocleidomastoideus, tiefe Nackenmuskulatur) wird infiltriert, die Projektionszone (Dermatom) gequaddelt und der Nervenaustrittspunkt des peripheren Nerven (z. B. N. occipitalis major) angespritzt (Abb. 5). Die Anwendung der TLA orientiert sich am Ort und der Art des Auftretens von Schmerzen (Projektionsschmerz, pseudoradikulärer Schmerz). Die TLA eignet sich gut zur Durchbrechung akuter Schmerzzustände (akuter Schiefhals, Zervikalmigräne) und zur begleitenden Therapie bei chronisch-reflektorischem Schmerzgeschehen. Zum Einsatz kommen Lokalanästhetika $(0,5-2 \%)$ ohne Vasokonstringens [5].

Die systemische Schmerztherapie richtet sich nach der Aktualität des Krankheitsbildes. Bei akuten Funktionsstörungen mit starker reflektorischer Verspannung der Muskulatur sollte durch antiphlo- 
gistisch-analgetische Vorbehandlung die Manualtherapie vorbereitet werden. Eine hochdosierte, kurzzeitige Gabe nicht-steroidaler Antirheumatika (NSAR) - bevorzugt werden sollten moderne Coxibe - kann mit klassischen Zyklooxygenasehemmern (z.B. ASS) kombiniert werden [34].

In der Langzeittherapie chronischer Funktionsstörungen (chronifizierte HWS-Weichteildistorsion, rheumatischer Formenkreis) ist eine minimal dosierte antiphlogistische Therapie (möglichst auch moderne Coxibe - z.B. Bextra ${ }^{\circledR}$ ) mit einer maximal dosierten analgetischen Komponente (auch unter Einsatz von Morphinen, THC-Analoga u.a. moderner Konzepte) zu kombinieren $[12,35]$. Der zusätzliche Einsatz eines TENS-Gerätes und von Entspannungstechniken (Atemtherapie, autogenes Training) ist hilfreich. Zum einen muss auf diesem Wege erreicht werden, dass der spinale Schmerzspeicher suffizient gelöscht wird [35] und zum anderen muss der Circulus vitiosus der schmerzinduzierten- und induzierenden Muskelverkürzung bei der segmentalen Störung durchbrochen werden [36]. Ohne Schmerzfreiheit ist keine weitere Therapie effizient möglich (s.o.) und die sekundären psychischen Störungen sind gebahnt. In der Behandlung der chronischen Schmerzzustände (vgl. 3.1.2) sollten bei der medikamentösen Therapie Myotonolytika berücksichtigt werden [28].

\subsection{Medizinische Trainingstherapie zur Sicherung des Langzeittherapieerfolges}

Die medizinische Trainingstherapie (MTT) dient der Stabilisierung des chirurgisch-konservativ erzielten Therapieerfolges nach komplexen Verletzungen oder soll bei kongenitaler oder habitueller Form- und Fehlstellung (Skoliose, Hypermobilität) die Rumpfmuskulatur aufbauen und kräftigen, um das Erkrankungspotenzial bei diesen Patienten zu vermindern [37]. Die Möglichkeiten der MTT sind sehr variabel und reichen von einer individualisierten Sporttherapie (z. B. Kieser-Training ${ }^{\circledR}$ ) über eine modifizierte, gerätegestützte Rückenmuskelaufbautherapie $\left(\right.$ FPZ-Konzept ${ }^{\circledR}$ ) [20] hin bis zu einzelnen Sportphysiotherapieeinheiten (mit Nordic Walking, Sportgymnastik usw.) und Selbstübungen [vgl. 38, 39]. MTT im besten Sinne dient der Prävention der Rezidiverkrankung oder der Stabilisierung des Therapieerfolges [40]. MTT-Konzepte müssen aber individualisiert auf das konstitutionelle Profil und das HWS-Erkrankungsmuster jedes einzelnen Patienten zugeschnitten werden, so dass es selten gelingt, vorhandene Programme (s. o.) „standardisiert“ anzuwenden.

\subsection{Begleitende Therapiemaßnahmen}

Begleitende Therapiemaßnahmen jenseits der medikamentösen Therapie und der medizinischen Trainingstherapie dienen zum einen der Vorbereitung manualmedizinischer Behandlung (z.B. Atemtechnik) [5], zum anderen sind sie für den Therapieerfolg essenziell (z.B. Psychotherapie bei posttraumatischem, chronischem Schmerzsyndrom, „Zervikalsyndrom“) [23,41]. Hierbei unterscheidet man im Einzelnen:

- Die Atemtechnik dient der Fazilitation therapeutischer Maßnahmen, um durch Ausnutzen der physiologischen Tonusänderungen der Muskulatur mit dem Atemrhythmus die Einzeltechniken der Mobilisation und Manipulation effizienter einzusetzen.
- Die Blickwendetechnik dient ebenfalls der Fazilitation therapeutischer Maßnahmen, da besonders im Bereich der HWS durch die Verschaltung optischer, vestibulospinaler u.a. Afferenzen Veränderungen des jeweiligen sensorischen Inputs sofort zu muskulären Reaktionen (Bahnung der Kopf- und Rumpfbewegung in Blickrichtung) mit nachfolgender Beeinflussung der Gelenkfunktionen führt. Zudem beeinflusst die Blickrichtung auch die Atmung (Blick nach oben/unten-Bahnung der Einatmung/Ausatmung). Maximale Blickwendungen wirken jedoch hemmend.

- Psychotherapeutisch-psychosomatisch begleitend sollten Patienten behandelt werden, wenn eine akute posttraumatische Belastungsstörung (PTBS) die spezifischen Therapiemaßnahmen infrage stellt [3] oder wenn chronische Schmerzen (bei schlechter Einstellbarkeit) oder sekundäre psychische Störungen (sekundäre, reaktive Depression, somatoforme Schmerzstörungen, Angst- und Anpassungsstörung) den Therapieprozess verzögern [28]. Dazu zählen auch das Vermitteln von Entspannungstechniken (z.B. autogenes Training). In diesem Zusammenhang wird in der Literatur gerne vom „Coping and counselling“ von Patient und Therapeut (d.h. Physiotherapeut, „Organmediziner“) gesprochen, um durch externe erfahrene Psychotherapeuten Barrieren gegenseitig abzubauen und ein tieferes Verständnis für die Entstehungsursachen der sekundären, psychischen Fehlentwicklung (Hauptprädiktor für deren Entstehung: unzureichende Schmerzeinstellung!) $\mathrm{zu}$ befördern $[8,9,14]$.

- Die Orthesenbehandlung (Standard: Miami J-Collar) [42] dient der kurzfristigen Ruhestellung (max. 12-24 Stunden) nach akutem Trauma oder der stabilisierenden Behandlung von Schwerstverletzten (z.B. Densfraktur). Sie ist ebenso hilfreich in der Rehaphase, um eine kurzfristige (stundenweise) muskuläre Entlastung nach intensiver Therapie/Training zu erzeugen. Längeres Tragen (über Tage) - vor allem nach Unfällen gilt als obsolet [13].

\section{Strukturell bedingte Funktionsstörungen der HWS und des kraniozervikalen Übergangs}

Insbesondere posttraumatische Folgezustände sind durch makrostrukturelle Veränderungen an der HWS und dem kraniozervikalen Übergang charakterisiert (z.B. Frakturen), die in erster Linie einer chirurgischen Therapie bedürfen [28]. Mikrostrukturelle Veränderungen (selten findet sich ein bildgebendes Korrelat) sind ebenfalls möglich (Myelonuntergang, Verkalkung von subligamentären Hämatomen, Myositis und Tendinose infolge Überdehnung), die selten chirurgisch, hauptsächlich manualmedizinisch-medikamentös behandelt werden [28].

Die strukturell bedingten Funktionsstörungen, zu denen auch postchirurgische Folgezustände, angeborene und chronisch-degenerative Veränderungen zählen, lassen sich kaum vollständig funktionswiederherstellend behandeln, als vielmehr kompensatorisch oder ggf. nur lindernd, symptomzentriert (Physiotherapie, Schmerzbehandlung!). Deshalb kann der nachfolgende Abschnitt nur einen Überblick geben, ohne wirklich alle Facetten dieses ausgedehnten Komplexes zu beleuchten. 
3.1 Posttraumatische Störungen der Wirbelsäule, des Kopfgelenkes, des Weichteil- und Bandapparates, der Bandscheiben und des Rückenmarkes

\subsubsection{Operative Therapie}

Die Grundsätze der operativen Behandlung von Strukturstörungen der Halswirbelsäule, seien sie bedingt durch Trauma, Tumor, Entzündung oder degenerative Veränderungen, beinhalten prinzipiell das gleiche Vorgehen nach [42]:

- Dekompression neuronaler Strukturen (Myelon oder Nervenwurzeln),

- Stabilisierung des betroffenen Bewegungssegmentes,

- Stellungskorrektur.

Es wird immer danach gestrebt, den Eingriff auf möglichst wenig Bewegungssegmente zu beschränken. Durch die bildgebenden Verfahren (CT, NMR), eine fachneurologische Untersuchung und Zusatzuntersuchungen (EMG-, MEP-, SSEP-Untersuchungen) gelingt in der Regel eine eindeutige Höhenlokalisation und segmentale Zuordnung [28].

\section{a) Obere Halswirbelsäule, Fraktur der Okzipitalkondylen}

Die Behandlung der Fraktur der Occipitalcondylen richtet sich nach dem Grad der Instabilität und der Dislokation der Fragmente. Neben dem Auftreten einer hohen Tetraplegie treten Läsionen der kaudalen Hirnnerven auf. Die neurologischen Defizite der Hirnnerven bilden sich in der Regel zurück. In Anlehnung an die Klassifikation von Anderson und Montesano [44] reicht beim Typ I die Therapie mit einer starren Zervikalstütze (Miami-J) für die Dauer von 6 Wochen aus. Dies gilt ebenso für den Typ II ohne atlanto-okzipitale Dislokation. Typ II mit atlanto-okzipitaler Dislokation und Typ III stehen nach Reposition die externe Fixation mit Halo-Jacket oder die C0/C2-Fusion von dorsal zur Verfügung.

\section{b) Fraktur des Atlasbogens}

Es existieren mehrere Frakturklassifikationen, die alle im Hinblick auf die daraus resultierende Therapie die Instabilität bzw. Stabilisierung berücksichtigen [vgl. 42]. Bei einem Bruch des vorderen oder hinteren Bogens ist eine Zervikalstütze für 6 Wochen anzulegen. Bei Brüchen des vorderen und des hinteren Bogens bei intaktem Ligamentum transversum atlantis ist auch hier eine Zervikalstütze oder eine Halofixation durchzuführen. Ist jedoch das Ligamentum transversum atlantis rupturiert, sollte hier eine C1/C2-Fusion zur Anwendung kommen. Liegt jedoch eine Tetraplegie vor, sollte eine primäre okzipito-zervikale Fusion CO/C2 durchgeführt werden. Eine rotatorische atlanto-axiale Dislokation wird nach Fielding und Hawkins [44] in 4 Typen unterschieden. Die nichttraumatische atlanto-axiale Rotationsluxation findet sich meist bei Mädchen, wobei sich in der Vorgeschichte eine juvenile rheumatoide Arthritis oder chronische, rezidivierende Infektionen der oberen Atemwege nachweisen lassen. Bei der traumatischen Genese liegt ein adäquates Trauma vor. Im frischen Stadium lässt sich die traumatische sowie die nichttraumatische Rotationsfehlstellung leicht reponieren mit anschließender Fixation im Halo-Jacket für 8-12 Wochen. Bei verspäteter Diagnosestellung ist eine länger andauernde Extensionsbehandlung erforderlich. In der Regel sind beim Typ I ohne Ruptur des Ligamentum transversum atlantis keine operativen Maßnahmen erforderlich.

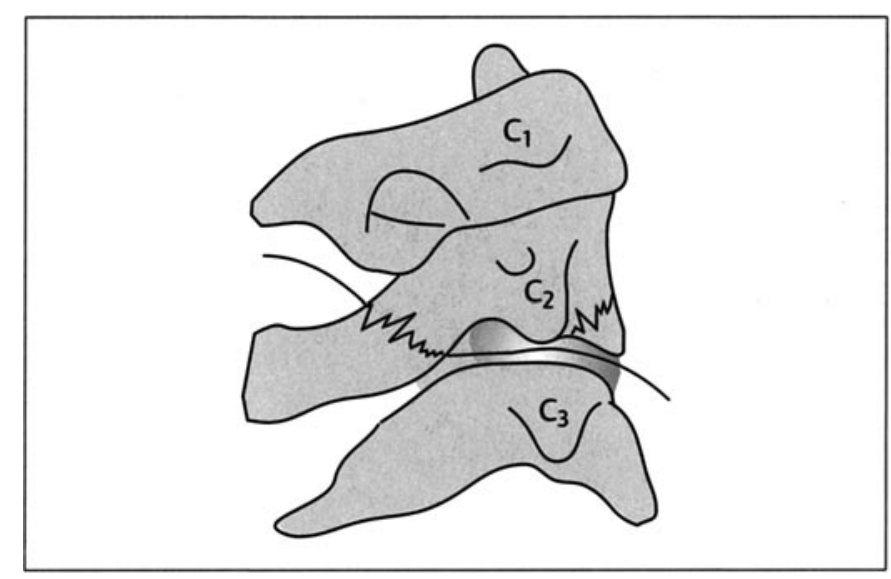

Abb. 6 Schematische Darstellung einer „hangman's fracture“. Aus: Ernst A, Herzog M, Seidl RO. Traumatologie des Kopf-Hals-Bereichs. Stuttgart, New York: Georg Thieme, 2004: 42, Abb. 5.4.

\section{c) C1-C2-Instabilität}

Hierzu gehören die isolierten Frakturen des Axis, des Dens axis sowie Kombinationsfrakturen des Atlas und Axis. Der Bruch des Bogens von C2 führt meist zu einer C2/C3-Instabilität.

Die Fraktur des Dens axis, die nach Anderson und D'Alonzo klassifiziert werden [42], werden entsprechend der Instabilität operativ oder konservativ behandelt. Beim Typ I - hierbei liegt lediglich eine Fraktur in der Densspitze vor - ist eine Ruhigstellung mit Zervikalstütze durchzuführen. Bei Frakturen vom Typ II, die bei konservativen Maßnahmen eine hohe Pseudarthroserate aufweisen, sollte eine operative Fusion durch Zugschraube von ventral durchgeführt werden [44]. Verläuft jedoch die Fraktur schräg von kranial-posterior nach kaudal-anterior, ist eine Zugschraube kontraindiziert. Hierbei empfiehlt sich der Einsatz einer Antigleitplatte. Die Fraktur vom Typ III - die Fraktur verläuft tief durch die Basis des Dens bzw. durch den Axis - sollte durch eine 12-wöchige Ruhigstellung mittels Halofixateur behandelt werden. Durch die Verwendung der Zugschraube ist nach knöcherner Ausheilung die Beweglichkeit im Bewegungssegment $\mathrm{C} 1 / \mathrm{C} 2$ nahezu uneingeschränkt möglich. Wenn jedoch relative Kontraindikationen zur Verwendung der Zugschraube vorliegen, bietet sich hier ebenfalls die Verschraubung nach Magerl von dorsal C2/C1 an [44], oder die dorsale Zerklage der Bögen C1/C2 mit Interposition eines Knochenspans nach Gallie oder Brooks mit entsprechender Bewegungseinschränkung der Re- und Inklination [43]. Die Kompression des Rückenmarkes im Bereich der oberen Halswirbelsäule bekommt durch die Nähe des Hirnstamms eine besondere Bedeutung. Die Struktur, die die komprimierende Kraft ausübt, sollte auf direktem Wege entfernt werden.

Die Behandlung der Verletzung des Bogens von HWK 2 ist abhängig von der Mitbeteiligung des Bandscheibenfaches C2/C3. Bei dieser unter dem Begriff ",hangman's fracture“(Abb. 6) bekannten traumatischen Spondylolisthese wird die isolierte Bogenfraktur von C2 konservativ durch eine starre Zervikalstütze (Miami J) [42] zur Ausheilung gebracht. Bei dem Zerreißen des hinteren Längsbandes und Erhalt des vorderen Längsbandes reicht eine Behandlung im Halo-Jacket aus. Bei der kompletten diskoligamentären Zerreißung im Bewegungssegment $\mathrm{C} 2 / \mathrm{C} 3$ ist eine ventrale Fusion C2/C3 notwendig. 


\section{d) Verfahren an der unteren Halswirbelsäule}

Über die Wahl des Zugangsweges (ventral oder dorsal) entscheidet ausschließlich der Ort des pathologischen Geschehens (wie liegt die komprimierende Struktur zum Myelon?) sowie die Möglichkeiten zur optimalen Wiederherstellung der Stabilität. Bei traumatischen diskoligamentären (zumeist monosegmentalen) Zerreißungen ist nach Resektion der zerstörten Bandscheibe eine Beckenkamminterposition mit anschließender ventraler Plattenosteosynthese erfolgreich. Bei Berstungsfrakturen mit begleitender ligamentärer Instabilität ist das Ziel der operativen Behandlung eine Wiederherstellung der anatomischen Achse. Hierdurch wird bereits ein wesentlicher Anteil der Dekompression erreicht. Zudem müssen der Spinalkanal enttrümmert und eine tragfähige vordere Säule hergestellt werden [44]. Zur Vermeidung sekundärer Reaktionen der Myelons erfolgt zusätzlich zur operativen eine pharmakologische „Dekompression“ mit einer hoch dosierten Methylprednisolongabe. In der NASCIS-II-Studie fanden sich Hinweise auf einen positiven Effekt, wenn innerhalb der ersten 8 Stunden mit der ultrahochdosierten Kortisontherapie entsprechend des NASCIS-II-Schemas begonnen wurde [45].

\subsubsection{Konservative Therapie}

Konservative Therapiemaßnahmen umfassen manualmedizinische und physikalische Verfahren, medikamentöse Zusatzbehandlung u.a. begleitende Therapiemaßnahmen (vgl. 2.2-2.4), die bereits in ihren Grundzügen dargestellt wurden (Abb.7). Das wichtigste klinische Anwendungsgebiet für konservative Therapiemaßnahmen ist die HWS-Weichteildistorsion (HWS-WTD, typisch: Auffahrunfall, untypisch: Überstreckung des Kopfes beim Aufschlagen des Rumpfes). Da an dieser Stelle keine diagnostischen, differenzialdiagnostischen, prognostischen oder sogar gutachterlichen Überlegungen zu diesem großen, zumeist kontrovers diskutierten Thema angestellt werden sollen, wird vorrangig auf das aktuelle Standardwerk zur HWS von Dvorak/Grob [28] verwiesen. Bei der HWS-WTD handelt es sich um den häufigsten Verletzungsmechanismus an der HWS (Abb. 8), bei dem es neben einer ausgeprägten Zug-/Druckbelastung der Weichteile und Gelenke der HWS und des kraniozervikalen Übergangs auch zu einem stumpfen Kopfanprall (an die Kopfstütze des PKW, ans Lenkrad, an den A-Holm, die Fensterscheibe; bei alternativen Unfallmechanismen auf eine harte Unterlage) kommen kann. In nur 5\% hinterlassen diese Verletzungen morphologisch fassbare Strukturverletzungen, die dann zumeist chirurgischoperativ behandelt werden (Densfraktur, Wirbelkörperfrakturen, Lig.-alare-Verletzung, traumatischer Bandscheibenvorfall, Aneurysma der A. vertebralis, intrazerebrale Einblutungen, Frontal/ Temporallappenödeme, Kalottenfraktur oder Fraktur der Schädelbasis) $[28,46] .85$ - 95\% aller HWS-WTD heilen innerhalb 3 Wochen folgenlos aus. Bei der Chronifizierung treten jedoch vielfältige klinische Beschwerden auf (s.u.) [47].

Bei Patienten mit akuter HWS-WTD sollte nach initialer Immobilisation (für Stunden, Miami J-Collar) und Schmerztherapie (vgl. 2.2.2) baldmöglichst die Mobilisationsbehandlung beginnen (passive/aktive Mobilisation, Verordnung von krankengymnastischer Übungsbehandlung, ggf. initial Kraniosakraltherapie) [32]. Das Therapieziel ist die Wiederherstellung des normalen HWSBewegungsumfangs und die zügige Rückkehr zum beruflichen und sozialen Alltag des Patienten [48]. Als spezifische Therapiemaßnahme bewährt sich hier insbesondere Vojta-Therapie [49].

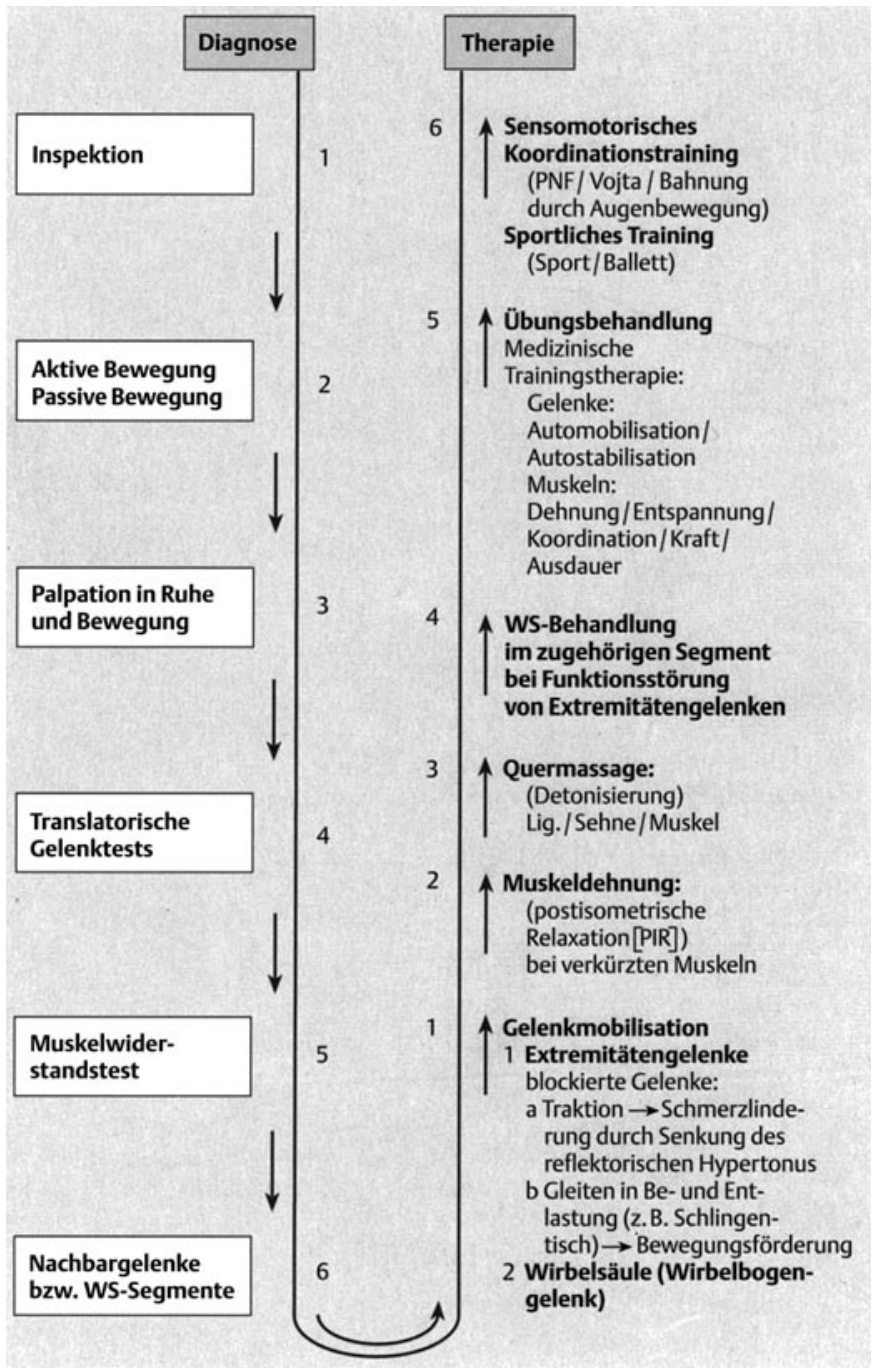

Abb. 7 Schematischer Ablauf der Therapieplanung bei Funktionsstörungen am Bewegungsapparat, aus: Ernst A, Meyer-Holz J, Weller E. Manuelle Medizin an der Halswirbelsäule. Stuttgart, New York: Georg Thieme, 1998: 36, Abb. 28.

Chronifizierte Folgezustände nach HWS-WTD (statistisch 5-15\% nach allen Unfällen) sind verknüpft mit chronischen Schmerzen, einer Bewegungseinschränkung und einer deutlichen Erhöhung des muskulären Binnentonus [48]. Hochrisikopatienten für eine Chronifizierung sind solche mit einer vorbestehenden Skoliose, einem Zweitunfall und mit initialen stärksten Schmerzen (neurogener Tiefenschmerz) $[14,30,50]$. Im Vordergrund der Behandlung stehen die Schmerztherapie (vgl. 2.2.2), vorsichtige Mobilisationsbehandlung, ggf. Kraniosakraltherapie und die Erarbeitung eines Selbstbehandlungskonzeptes (Übungen, Entspannungstherapie, physikalische Therapie, MTT - sofern möglich) $[5,13,28,51]$.

Ein Sonderfall stellt die Lig.-alare-Beteiligung (Abb. 9) bei durchgemachter HWS-WTD dar [52]. Diese Patienten sind hochgradig bewegungseingeschränkt im Kopfgelenksbereich, hochgradig erschütterungs- und schmerzempfindlich und bedürfen eines speziellen, primär schmerzorientierten Behandlungskonzeptes bei gleichzeitiger, hochdosierter Kortisongabe zur Verhinderung neuronaler Schäden (vgl. 3.1.1) [45]. 


\section{Phase I}

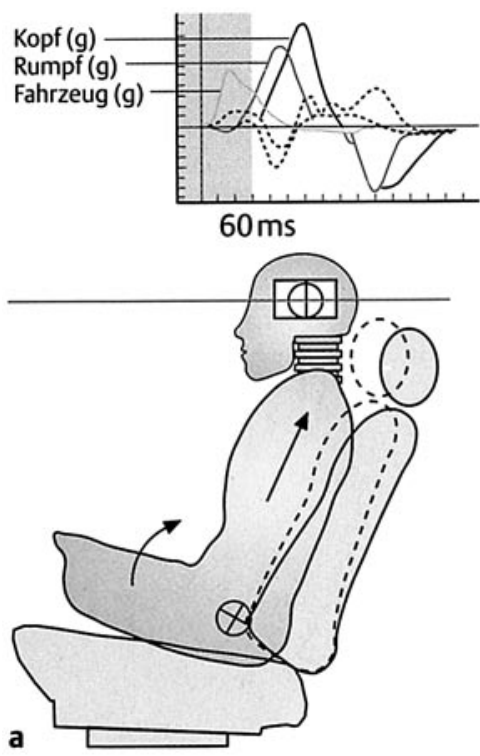

\section{Phase III}

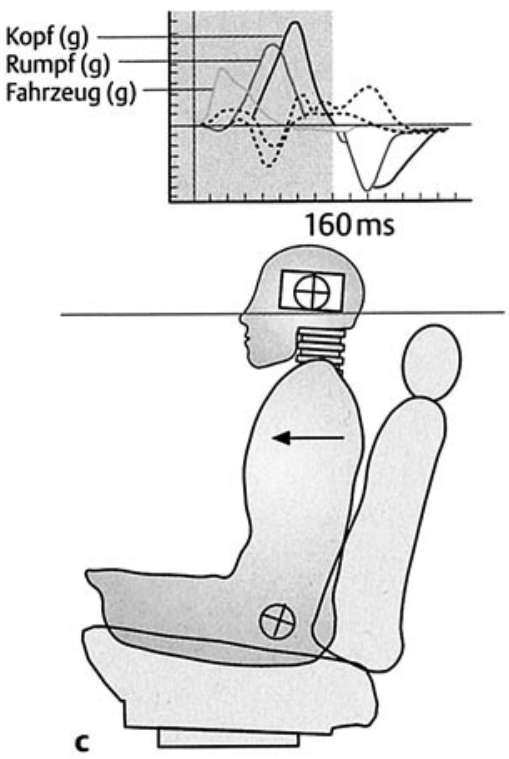

Phase II

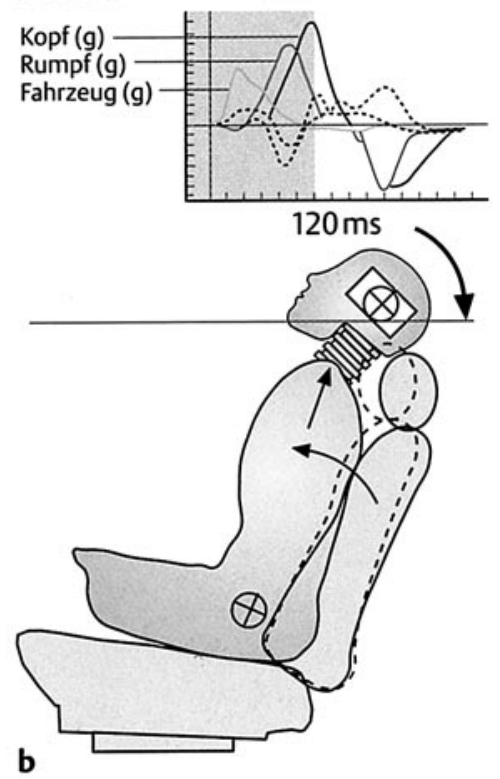

Phase IV

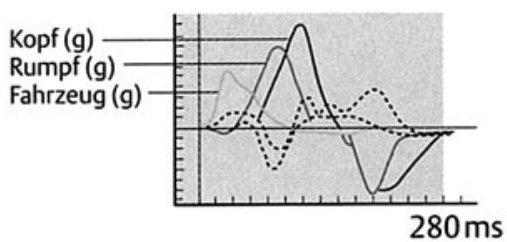

Abb. 8 Typischer 4-phasiger Pathomechanismus im Rahmen einer Beschleunigungsverletzung, aus: Ernst A, Herzog M, Seidl RO. Traumatologie des Kopf-Hals-Bereichs. Stuttgart, New York: Georg Thieme, 2004: 43, Abb. 5.5.

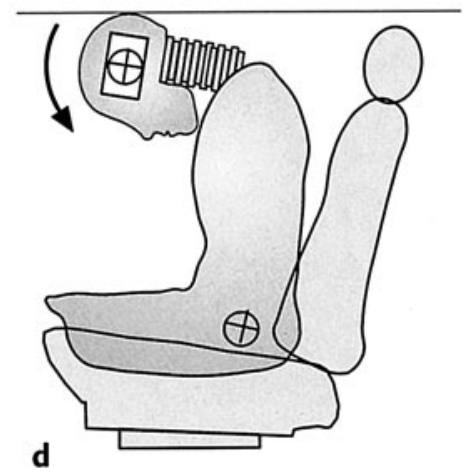

3.2

\section{Folgezustände nach tumorchirurgischen Eingriffen oder iatrogene Störungen der HWS und des kranio- zervikalen Übergangs}

Tumorchirurgische Eingriffe an den Halsweichteilen (z.B. Neck dissection) infolge der Primärtumorentfernung hinterlassen stärkere Vernarbungen, Muskel/Faszienverklebungen, isolierte Weichteil-, Muskel- und Nervenverluste, die zu unterschiedlich stark ausgeprägten Funktionsstörungen führen. Echte wiederherstellende Verfahren im Sinne einer Restitutio ad integrum sind deshalb kaum erfolgreich durchführbar. Eine Ausnahme bildet der chirurgische Transfer der Mm. levator scapulae et rhomboidei zur funktionellen Wiederherstellung der Schulterbeweglichkeit bei N.-accessorius-Parese [53]. Im Vordergrund stehen deshalb die Schmerzbehandlung (vgl. 2.2.2), die Verbesserung der Beweglichkeit (vor allem der Schulter-Arm-Beweglichkeit) der narbig und radiodermatitisch beeinträchtigten Haut, Unter- haut und Restmuskulatur (vgl. 2.2) und die Verbesserung des Lymphabflusses [5,34]. Die beiden vorgenannten Aspekte sind bereits ausführlich diskutiert worden. Der Lymphabfluss sollte durch Lymphdrainage verbessert werden.

Isolierte Narben und z.T. Muskeluntergänge der tiefen Nackenmuskeln nach Kraniotomien (retrosigmoidaler bzw. subokzipitaler Zugang) können einen migränoiden Kopfschmerz (Migraine cervicale) und selten eine isolierte Kopfgelenksinstabilität infolge muskulärer Dysbalance verursachen. Die Behandlung sollte symptombezogen (Migränetherapie, Muskelaufbau und MTT) verlaufen, wobei ein stundenweises Orthesentragen häufig erforderlich ist (vor allem bei akut einsetzender Zervikalmigräne). 


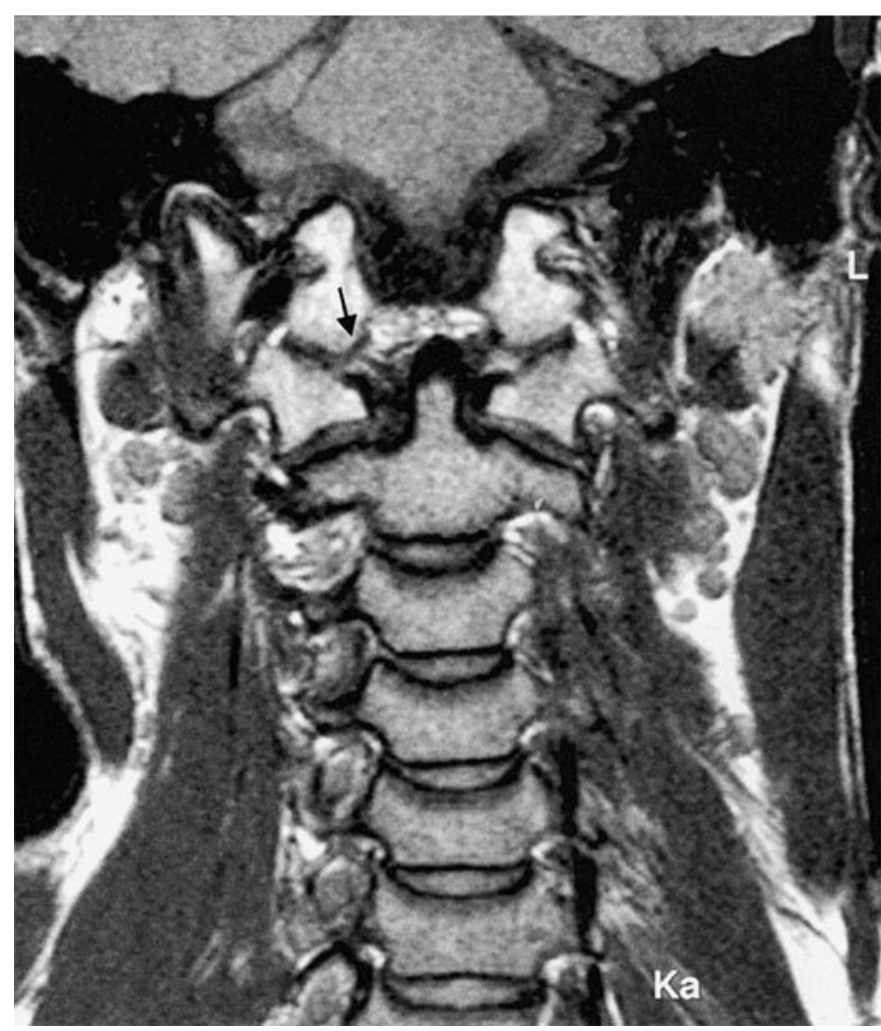

Abb. 9 Verletzung der Ligg. alaria $(\downarrow)$ nach einer Beschleunigungsverletzung (MRT-Aufnahme, Institut für Radiologie im ukb, Direktor: PD Dr. Mutze). Aus: Ernst A, Herzog M, Seidl RO. Traumatologie des KopfHals-Bereichs. Stuttgart, New York: Georg Thieme, 2004: 44, Abb. 5.6.

\section{Angeborene, chronisch-degenerative und andere Funktionsstörungen der HWS und des kranio- zervikalen Übergangs}

Die wichtigsten Determinanten für einen verzögerten Heilungsverlauf nach HWS-Trauma bzw. für häufig rezidivierende akute Funktionsstörungen (ob im Gelenk oder der Muskulatur) sind Wirbelsäulenskoliosierungen. Skoliosierungen oder Kyphoskoliosierungen erhöhen das Erkrankungspotenzial für Wirbelsäulenerkrankungen und insbesondere für Funktionsstörungen der HWS beträchtlich [28]. Insbesondere eine vestibulospinale (posturale) Unsicherheit mit verstärkten Körperschwankungen und subjektivem Schwindelgefühl wurden signifikant häufiger bei Skoliotikern nachgewiesen $[54,55]$ und ähneln darin Patienten mit einer chronifizierten HWS-WTD [56], bei denen jedoch die zugrunde liegende muskuläre Dysbalance Traumafolge ist. Allein deshalb raten viele Autoren zu einem vorbeugenden Muskelaufbautraining (MTT, vgl. 2.3) bei diesen Patienten, um durch eine gut kompensierte (und damit kompensierende) paraspinale Muskulatur akute Funktionsstörungen $\mathrm{zu}$ verhindern $[20,39,40]$. Ähnliches gilt für die allgemeine Hypermobilität, die zu einem unphysiologisch weiten Gelenkausschlag führt. Bei dieser Konstellation empfiehlt sich ebenfalls jahrelanges Muskelaufbautraining, um das Verletzungspotenzial zu verringern $[28,39]$. Zudem besteht bei diesen Patienten eine relative Kontraindikation für Manipulationsbehandlungen, insbesondere an der HWS (Verstärkung der Hypermobilität und damit Destabilisierung der Wirbelsäule mit subjektiver, posturaler Unsicherheit) [5].
Der enge Spinalkanal und neuroforaminale Stenosen führen zumeist zu starken, bewegungsabhängigen (radikulären) Schmerzen, dann Par- und Anästhesien und später zur Kraftlosigkeit. Der motorische Ausfall und das Ausmaß der Stenose bestimmt das Ausmaß und den Zeitpunkt der operativen Behandlung (vgl. 3.1.1) [28]. Im Initialstadium bringen manualmedizinische Behandlung, medikamentöse Zusatzbehandlung und ggf. Facetteninfiltrationen [57] eine symptomatische Reduktion der Schmerzen.

Strukturelle Veränderungen (z.B. Osteochondrose, Spondylose, Blockwirbelbildung, Bandscheibenprolaps) müssen nicht unbedingt ein Krankheitspotenzial beinhalten. Der Therapiebedarf richtet sich deshalb immer nach dem Ausmaß der klinisch relevanten Beschwerden [28].

Die echten rheumatischen oder rheumatoiden Erkrankungen, die die HWS mitbetreffen sowie Erkrankungen aus dem immunologischen Formenkreis (z.B. M. Bechterew) sind in der Regel komplexe Krankheitsabläufe mit spezifischen Therapiestandards, so dass an dieser Stelle auf eine ausführliche Darstellung verzichtet werden muss [zur Übersicht vgl. 28]. Die Leitsymptome bewegungsabhängiger Schmerz und Bewegungseinschränkung lassen sich aber parallel - wie bereits beschrieben - behandeln (vgl. $2.2-2.4)$.

\section{$4 \quad$ Rationale Therapieplanung nach Leitsymptomen}

Für den HNO-Arzt bedeutsam sind Leitsymptome, die sich als vertebragen einordnen lassen. Das wichtigste Leitsymptom ist der (unsystematische, als Unsicherheitsgefühl angegebene) Schwindel, der sich zumeist auf eine Kopfgelenksblockierung oder eine posturale Instabilität mit erhöhter Körperschwankungsbreite (infolge chronifizierter muskulärer Fehlfunktion, z.B. nach HWS-WTD) zurückführen lässt $[56,58,59]$. Er spricht gut auf einzelne manualmedizinische Maßnahmen und ein zusätzliches Koordinationstraining zur muskulären Stabilisierung des vestibulospinalen Anteils des Gleichgewichtssystems an [60]. Hier muss differenzialtherapeutisch die Otolithenfunktionsstörung nach stumpfem Kopfanprall als wichtigste, klinisch ähnlich erscheinende Erkrankung abgegrenzt werden $[59,61]$. Die Otolithenfunktionsstörung (sakkulär oder utrikulär bedingt) entsteht zumeist durch den begleitenden Kopfanprall (z.B. an die Kopfstütze bei der HWS-WTD) bei Beschleunigungsverletzungen an der HWS/dem kraniozervikalen Übergang. Weitere, zumeist durch den Kopfanprall an die Kopfstütze oder andere Fahrzeuginnenteile (z.B. den A-Holm) ausgelöste Schwindelformen ähneln in ihrer Systematik denen nach gedecktem SHT und sind in der Regel gut therapeutisch beherrschbar, erfordern jedoch ein hohes Maß an diagnostischer Power zur Aufdeckung der Störung [zur Übersicht vgl. 59,60].

Hörstörungen und Tinnitus (tieffrequent) können ebenso akut die Folge einer Gelenkblockade in der oberen HWS (oder dem Kopfgelenk) sein. Sie sprechen gut auf Manipulationsbehandlungen an [62]. Abzugrenzen sind hiervon vor allem posttraumatische, zentrale Hörstörungen nach HWS-WTD (z. B. mit Kopfanprall an die Kopfstütze) [63], bei denen es in der Folge des Kopfanpralls zu axonalen Schäden in der zentralen Hörbahn mit charakteristi- 
schen Ausfällen in der objektiv-audiologischen Funktionsdiagnostik kommt und die durch die Leitsymptome Tinnitus und Hyperakusis gekennzeichnet sind [63].

Schluckstörungen und Globusgefühl (bzw. pseudopektanginöse Beschwerden) können die Folge von Bandscheibenprotrusionen, osteophytischen oder osteochondrotischen Anbauten oder Gelenkblockaden in der tiefen HWS sein. Sie sprechen meist gut auf Ma- nualmedizin an $[64,65]$, in seltenen Fällen ist eine neurophysiologisch angelegte, fazio-orale Trakttherapie (FOTT) oder ein chirurgisches Vorgehen erforderlich [66].

Die Otalgie ist häufig Ausdruck einer kraniomandibulären Dysfunktion (CMS) und bedarf in der Regel einer eingehenden zahnärztlich-funktionsdiagnostischen Abklärung [62,67,68]. 


\section{Literatur}

${ }^{1}$ Neuhuber WL. Besonderheiten der Innervation des Kopf-Hals-Bereichs. Orthopäde 1998; 27: 794-801

2 Wolff H-D (Hrsg.). Die Sonderstellung des Kopfgelenkbereichs. Berlin, Heidelberg, New York, London, Paris, Tokyo: Springer, 1988

${ }^{3}$ Hülse M, Neuhuber WL, Wolff HD (Hrsg.). Der kranio-zervikale Übergang. Berlin, Heidelberg, New York, Barcelona, Budapest, Hongkong, London, Mailand, Paris, Santa Clara, Singapur, Tokio: Springer, 1998

${ }^{4}$ Wolff H-D. Neurophysiologische Aspekte des Bewegungssystems. Berlin, Heidelberg, New York, Barcelona, Budapest, Hong Kong, London, Mailand, Paris, Santa Clara, Singapur, Tokyo: Springer, 1996

${ }^{5}$ Ernst A, Meyer-Holz J, Weller E. Manuelle Medizin an der HWS. Stuttgart: Thieme, 1998

${ }^{6}$ Lewit K. Manuelle Medizin. Leipzig, Berlin, Heidelberg: Johann Ambrosius Barth, 1992

7 Patel RV, DeLong W Jr, Vresilovic EJ. Evaluation and treatment of spinal injuries in the patient with polytrauma. Clin Orthop 2004; 422: $43-54$

${ }^{8}$ Sonderlund A, Lindberg P. Whiplash-associated disorders-predicting disability from a process-oriented perspective of coping. Clin Rehabil 2003; 17: $101-107$

${ }^{9}$ Mayou R, Bryant B. Psychiatry of whiplash neck injury. Br J Psychiatry 2002; 180: $441-448$

${ }^{10}$ Murray J, Ehlers A, Mayou RA. Dissociation and post-traumatic stress disorders: two prospective studies of road traffic accident survivors. Br J Psychiatry 2002; 180: $363-368$

${ }^{11}$ Antepohl W, Kiviloog L, Andersson J, Gerdle B. Cognitive impairment in patients with chronic whiplash-associated disorder - A matched control study. Neuro Rehabilitation 2003; 18: $307-315$

${ }^{12}$ Curatolo M, Petersen-Felix S, Arendt-Nielsen L, Giani C, Zbinden AM, Radanov BP. Central hypersensivity in chronic pain after whiplash injury. The Clinical Journal of Pain 2001; 17: 306-315

${ }^{13}$ Foreman SM, Croft AC. Whiplash Injuries. Baltimore: Williams \& Wilkins, 1995

14 Olivegren H, Jerkvall N, Hagström Y, Carlsson J. The long-term prognosis of whiplash-associated disorders (WAD). Eur Spine J 1999; 8: $366-370$

15 Otte A, Ettlin T, Nitzsche EU, Wachter K, Hoegerle S, Simon GH, Moser E, Mueller-Brand J. PET and SPECT in whiplash syndrome: a new approach to the forgotten brain? J Neurol Neurosurg Psychiatry 1997; 63: $368-372$

${ }^{16}$ Freitag P, Greenlee MW, Wachter K, Ettlin T, Radue EW. FMRI response during visual motion stimulation in patients with late whiplash syndrome. Neurorehabl Neural Repair 2001; 15: 31 - 37

${ }^{17}$ Biedermann H. Das KISS-Syndrom der Neugeborenen und Kleinkinder. Manuelle Med 1993; 30: 91 - 107

18 Coenen W. Manualmedizinische Diagnostik und Therapie bei Säuglingen. Manuelle Med 1996; 34: 108 - 114

${ }^{19}$ Coenen W. Die sensomotorische Integrationsstörung. Manuelle Med 1996; 34: 141 - 146

${ }^{20}$ Denner A. Analyse und Training der wirbelsäulenstabilisierenden Muskulatur. Berlin, Heidelberg, New York, Barcelona, Budapest, Hongkong, London, Mailand, Paris, Singapur, Tokio: Springer: 1998

${ }^{21}$ Eder M, Tilscher H. Chirotherapie. Stuttgart: Hippokrates, 1995

22 Schneider W, Dvorák J, Dvorák V, Tritschler T. Manuelle Medizin. Stuttgart, New York: Georg Thieme, 1986

${ }^{23}$ Müller K, Kreutzfeldt A, Schwesig R, Müller-Pfeil J, Bandemer-Greulich U, Schreiber B, Bahrke U, Fikentscher E. Hypermobilität und chronischer Rückenschmerz. Manuelle Medizin 2003; 41: 105-109

${ }^{24}$ Sachse J. Die Formen der Hypermobilität und ihre klinische Einordnung. Manuelle Medizin 2004; 42: 27 - 32

${ }^{25}$ Chaitow L. Neuromuskuläre Techniken in der Manuellen Medizin und Osteopathie. München, Jena: Urban \& Fischer, 2002

${ }^{26}$ Upledger JE, Vredevoogd JD. Lehrbuch der Kraniosakraltherapie. Heidelberg: Karl F. Haug, 1996

27 McCroy DC, Penzien DB, Hasselblad V, Gray RN. Verhaltenstherapie und physikalische Behandlungen bei Spannungs- und zervikogenen Kopfschmerzen. Manuelle Medizin 2001; 39: 177-181

${ }^{28}$ Dvorak J, Grob D. Halswirbelsäule. Stuttgart: Thieme, 1999

${ }^{29}$ Feng FL, Schofferman J. Chronic neck pain and cervicogenic headache. Curr Treat Options Neurol 2003; 5: 493-498

${ }^{30}$ Suissa S, Harder S, Veilleux M. The relation between initial symptoms and signs and the prognosis of whiplash. Eur Spine J 2001; 10: 44-49
${ }^{31}$ Kasch H, Stengaard-Petersen K, Arendt-Nielsen L, Jensen TS. Headache, neck pain, and neck mobility after acute whiplash injury. Spine 2001; 26: 1246-1251

32 Spitzer WO, Skovron ML, Salmi LR et al. Scientific monograph of the Quebec Task Force on whiplash-associated disorders: redefining whiplash and its management. Spine 1995; 20 (Suppl 8): S1 -S73

33 Tilscher H, Eder M. Infiltrationstherapie. Stuttgart: Hippokrates, 1991

${ }^{34}$ Miehle W. Medikamentöse Therapie rheumatischer Erkrankungen. Stuttgart, New York: Georg Thieme Verlag, 2000

35 Banic B, Petersen-Felix S, Andersen OK, Radanov BP, Villiger PM, Arendt-Nielsen L, Curatolo M. Evidence for spinal cord hypersensitivity in chronic pain after whiplash injury and in fibromyalgia. Pain 2004; 107: 7-15

${ }^{36}$ Carette S. Whiplash injury and the chronic neck pain. N Engl J Med 1994; 330: $1083-1084$

${ }^{37}$ Günther P, Seidel EJ, Wick Ch. Manuelle Medizin und medizinische Trainingstherapie. Manuelle Therapie 2003; 41: 309-312

${ }^{38}$ Kisner C, Colby LA. Therapeutic execise. Foundations and techniques. Philadelphia: F. A. Davis Company, 1996

39 Tilscher H, Eder M. Wirbelsäulenschule aus ganzheitlicher Sicht. Stuttgart: Hippokrates, 1999

${ }^{40}$ Ylinen J, Takala EP, Nykänen A, Häkkinen A, Mälkia E, Pohjalainen T, Karppi SL, Kautiainen SL, Airaksinen O. Aktives Nackenmuskeltraining in der Behandlung chronischer Nackenschmerzen bei Frauen. Manuelle Medzin 2003; 41: 491 - 499

${ }^{41}$ Sullivan MJ, Hall E, Bartolacci R, Sullivan ME, Adams H. Perceived cognitive deficits, emotional distress and disability following whiplash injury. Pain Res Manag 2002; 7: 120-126

42 Ernst A, Herzog M, Seidl RO. Traumatologie des Kopf-Hals-Bereichs. Stuttgart, New York: Thieme, 2004

${ }^{43}$ Levine A. Spine trauma. Philadelphia: Saunders, 1998

${ }^{44}$ Tscherne H. Tscherne Unfallchirurgie - Wirbelsäule. Berlin, Heidelberg, New York: Springer, 1998

${ }^{45} \mathrm{NIH}$ Consensus Statement on rehabilitation of persons with traumatic brain injury. JAMA 1999; 282: $974-983$

${ }^{46}$ Saternus K-S. Die Verletzungen von Halswirbelsäule und Halsweichteilen. In: Junghanns H (Hrsg). Die Wirbelsäule in Forschung und Praxis. Bd. 84. Stuttgart: Hippokrates, 1979

${ }^{47}$ Freeman MD, Croft AC, Rossignol AM. Whiplash associated disorders: Redefining whiplash and its management by the Quebec Task Force. Spine 1998; 23: $1043-1049$

${ }^{48}$ Hartling L, Brison RJ, Ardern C, Pickett W. Prognostic value of the Quebec Classification of Whiplash Associated Orders. Spine 2001; 26: $36-41$

49 Vojta V, Peters A. Das Vojta-Prinzip. Berlin, Heidelberg, New York: Springer, 1997

${ }^{50}$ Khan S, Bannister G, Gargan M, Asopa V, Edwards A. Prognosis following a second whiplash injury. Injury, Int J Care Injured 2000; 31: 249-251

51 Pioch E, Seidel W. Manuelle Medizin in der Behandlung chronischer Schmerzsyndrome. Manuelle Medizin 2003; 41: $92-104$

52 Schröter T, Parais S, Arndt H, Ernst A, Mutze S. MRT der Ligg. alaria symptomatischer Patienten nach HWS-Distorsionstrauma. Trauma \& Berufskrankh 2002; 4: 224-229

53 Romero I, Gerber C. Levator scapulae and rhomboid transfer for paralysis of trapetius. J Bone Joint Surg 2003; 85: 1141 - 1145

${ }^{54}$ Chen PQ, Wang JL, Tsuang YH, Liao TL, Huang PI, Hang YS. The postural stability control and gait pattern of idiopathic scoliosis adolescents. Clin Biomech 1998; 13 (Suppl 1): 52-58

${ }^{55}$ Manzoni D, Miele F. Vestibular mechanism involved in idiopathic scoliosis. Arch Ital Biol 2002; 140: 67-80

${ }^{56}$ Sjostrom H, Allum JHJ, Carpenter MG et al. Trunk sway measures of postural stability during clinical balance tests in patients with chronic whiplash injury symptoms. Spine 2003; 28: 1725-1734

57 Slipman CW, Lipetz JS, DePalma MJ, Jackson HB. Therapeutic selective nerve root block in the nonsurgical treatment of traumatically induced cervical spondylotic radiating pain. Am J Phys Med Rehabil 2004; 83 (6): $446-454$

${ }^{58}$ Allum JHJ, Zamani F, Aitkin AL, Ernst A. Differences between trunk sway characteristics on a foam support surface and on the Equitest ankle-sway-referenced support surface. Gait \& Posture 2002; 16: $264-270$

${ }^{59}$ Ernst A, Basta D, Todt I, Seidl RO, Scherer H, Clarke A. Management of posttraumatic vertigo. Otol Head \& Neck Surg 2004; 131: 225-229 
${ }^{60}$ Ernst A, Seidl RO, Nölle C, Pudszuhn A, Ganslmeier A, Ekkernkamp A, Mutze S. Hör- und Gleichgewichtsstörungen nach Kopfanpralltraumen. Trauma Berufskrankh 2001; 3: 27-31

${ }^{61}$ Vibert D, Hausler R. Acute peripheral vestibular deficits after whiplash injuries. Ann Otol Rhinol Laryngol 2003; 112: 246-251

${ }^{62}$ Biesinger E, Heiden C. Ohrenschmerz und Funktionsstörungen der HWS. HNO 1994; 42: $207-213$

${ }^{63}$ Nölle D, Todt I, Ernst A. Pathophysiological changes of the central auditory pathway after blunt trauma of the head. J Neurotrauma 2003; 21: $251-258$

${ }^{64}$ Biesinger E, Schrader M, Weber BP. Die Osteochondrose der HWS als Ursache von Globusgefühl und Dysphagie. HNO 1989; 37: 33 - 35
${ }^{65}$ Klett R, Röhrl A. Nächtliche und belastungsbedingte pektanginöse Beschwerden durch vertebragene Dysfuntkionen. Manuelle Medizin 2001; 39: $17-19$

${ }^{66}$ Nusser-Müller-Busch R (Hrsg). Die Therapie des facio-oralen Trakts. Berlin, Heidelberg, New York, Hongkong, London, Mailand, Paris, Tokio: Springer, 2004

${ }^{67}$ Freesmeyer WB. Zahnärztliche Funktionstherapie. München, Wien: Carl Hanser, 1993

${ }^{68}$ Kopp S, Friedrichs A, Pfaff G, Langbein U. Beeinflussung des funktionellen Bewegungsraumes von Hals-, Brust- und Lendenwirbelsäule durch Aufbissbehelfe. Manuelle Medizin 2003; 41: 39-51 